\title{
Thoughts on Wackers' call for a standard unit of defect size
}

\author{
Denny D. Watson, Ph.D. ${ }^{\mathrm{a}}$ \\ a University of Virginia Health System, Charlottesville, VA \\ Received Feb 13, 2014; accepted Feb 13, 2014 \\ doi: $10.1007 /$ s12350-014-9875-7
}

\section{See related article, pp. 673-681}

As we try to pass along advice to the next generation of those who will engage in medical science, I endorse Dr. Wackers' plea for reproducible standards for measurements of myocardial perfusion. Standards, from bearing sizes to protocols for Internet communication, have certainly enabled the industrial revolution and propelled modern technology. By comparison, medical science is far behind. Beyond the request for standardized measurements, I also believe that more careful thought about what we are measuring will be important.

Useful standards can be constructed only from welldefined and reproducible quantities. Volumes can be precisely defined, so measurements of end-diastolic and end-systolic volume, EF and in principle of myocardial mass can be constructed and tested for accuracy and precision based on a well-defined ground truth. Perfusion measurements are more elusive. Absolute myocardial blood flow and flow reserve can be precisely defined, but are probably not yet in the realm of being routinely measured and diagnostically interpreted. Our present quantitative methods can attempt to estimate the fraction of myocardium that is ischemic (ischemic volume-or infarct volume if we can correctly differentiate). But suppose we measure " $20 \%$ ischemic myocardium." Twenty percent of the myocardium may be severely ischemic at rest or mildly ischemic at 12 METs and that would span a huge swath of diagnostic and prognostic territory. The stress and rest score method is a hopefully rational albeit arbitrary attempt to factor in severity of flow reduction with the compromised volume and represent more of an "ischemic burden,', but is semiquantitative and curiously difficult to precisely define. The error of estimation of this quantity is most important but particularly difficult to determine. Knowing the error of estimate is critical to determine if the numbers being measured can successfully classify a single patient as opposed to the average of a patient population.

Thus, we measure something, without understanding precisely what it is, then correlate it with pathology or outcome and pronounce that it is now a "validated", standard measurement. Then, we ask that all other measurements be calibrated to the validated "standard.', This is actually not the path that propels modern technology. We need more careful thought and more precise definitions of what we are trying to measure: enough so that we have some level of ground truth by which to determine the accuracy and precision of the various quantitative methods, and some basic understanding of the physiological significance of the measurement. Population correlations alone cannot establish an adequate basis for reliable standardized measurements.

So, from an engineering viewpoint, I agree with the need for better standards, and would additionally encourage the next generation to get past simple correlations and do some hard-headed scientific thinking about what is being measured and what needs to be measured and standardized.
Reprint requests: Denny D. Watson, Ph.D., University of Virginia Health System, 103 Woodhurst Court, Charlottesville, VA 22901; denny@virginia.edu.

J Nucl Cardiol 2014;21:685.

$1071-3581 / \$ 34.00$

Copyright (C) 2014 American Society of Nuclear Cardiology. 